\title{
MICRO RF TAGS FOR MEDICAL IMAGING
}

\author{
J.J. Bernstein ${ }^{1}$, M.G. Bancu ${ }^{1}$, R. Ciocan ${ }^{2}$, R.E. Lenkinski ${ }^{2}$, R. Marquis ${ }^{2}$, A. Ivanishev ${ }^{2}$, and J.V. Frangioni ${ }^{2}$ \\ ${ }^{1}$ The Charles Stark Draper Laboratory, Cambridge, Massachusetts, USA \\ ${ }^{2}$ Beth Israel Deaconess Medical Center, Boston, Massachusetts, USA
}

\begin{abstract}
This paper presents Micro Resonant Devices (MRD's) which are visible in MRI scans. These LC resonators are fabricated using electroplated spiral inductors combined with thin-film $\mathrm{Ta}_{2} \mathrm{O}_{5}$ capacitors, formed by a novel oxy-nitride plasma oxidation process. Arrays of resonators were fabricated with diameters of $0.3,0.5$ and $1 \mathrm{~mm}$. To compensate for process variations, resonators were fabricated with parametrically varying capacitor areas. In this study, we characterize the first such devices for their detectability as a function of size, in saltcontaining aqueous media and in vivo, using a clinical MRI scanner and a scanning UHF microscope.
\end{abstract}

\section{INTRODUCTION}

\section{Magnetic Resonant Imaging}

Magnetic Resonant Imaging (MRI) works by imaging the density of hydrogen nuclei, as well as variations in the spin relaxation times in the presence of a strong magnetic field [1]. The spin energy states of a hydrogen nucleus are split in a magnetic field, creating a small net magnetic moment. Transitions between the two states occur at the Larmor frequency which is proportional to the magnetic field. The classical interpretation of nuclear spin in a magnetic field gives a precession frequency equal to the Larmor frequency, which is a useful way of visualizing the magnetic moments in an MRI sample. In a 3T field, the Larmor frequency is $127.7 \mathrm{MHz}$.

Most MRI contrast agents perturb the RF B1 field or the spin relaxation times $\mathrm{T} 1$ and $\mathrm{T} 2$ [1], using elements with unpaired spins which can exchange energy with the proton magnetic spins. Examples are transition metal ions $\mathrm{Fe}^{3+}, \mathrm{Mn}^{2+}$, and $\mathrm{Gd}^{3+}$. Other contrast agents consist of non-stoichiometric iron oxide nano-particles called MIONs [2].

The long term goal of this research is to detect a single device, in a single cell, anywhere in a living subject. This will require tags on the order of $10 \mu \mathrm{m}$ in size, capable of being endocytosed by cells. The devices presented here are a first step towards the tiny tags which can be inserted in single cells.

In this work, we present a novel RF MEMS tag which is visible in an MRI because it is resonant at the Larmor frequency. It can therefore exchange energy with the hydrogen nuclei and perturb the RF (B1) field and the spin relaxation times. The device consists of a spiral inductor and a thin film capacitor. The inductor also serves as an antenna, coupling to the external RF pulses and the nearby protons.

\section{DESIGN OF MICRO RESONANT DEVICES}

The goal was to fabricate Micro-Resonant Devices (MRD's) with a resonant frequency of $127.7 \mathrm{MHz}$. The process to make the capacitor dielectrics was developed simultaneously with the spiral inductors, and the process variability was unknown. Hence, the mask set was created with an array of parametrically varied resonators to cover the frequency range from 0.6 to $1.5 \mathrm{X}$ the Larmor frequency (77 MHz to $190 \mathrm{MHz}$ ) at 3\% frequency intervals. Inductors were constrained to 3 sizes: $0.3 \mathrm{~mm}, 0.5$ $\mathrm{mm}$ and $1.0 \mathrm{~mm}$. For each inductance size, the capacitor areas were varied to achieve the desired resonant frequency range. While this technique yields only a few devices resonant near the desired frequency, it was designed to yield some good resonators in the presence of unknown process variations in capacitance or inductance.

\section{Spiral Inductors and Capacitors}

Calculations of lumped spiral inductance were made using the modified Wheeler formula and checked with the current sheet formula [3]. These formulas agreed within a few $\%$. The skin depth $\delta$ for $\mathrm{Cu}$ is 5.8 microns at $127.7 \mathrm{MHz}$. The inductor trace width was kept $<2 \delta$ to efficiently use the $\mathrm{Cu}$ in the traces.

The capacitors were placed either at the inner or outer diameter of the spiral inductor, leaving an opening free of metal at the inductor center to allow free passage of magnetic flux. A simple lumped capacitor element was assumed.

\section{FABRICATION Capacitors}

To achieve the smallest possible LC resonator with the correct resonant frequency, capacitors with a high capacitance/area are required. Currently such dielectric films are being used as gate dielectrics in advanced MOSFETs. Atomic Layer Deposition (ALD) is used to deposit discrete monolayers of oxide films such as $\mathrm{HfO}_{2}$ and $\mathrm{Ta}_{2} \mathrm{O}_{5}$. A metaldielectric-metal capacitor (not MOS) is required for our tags, hence we chose a plasma oxidation process on a thin film of Ta, rather than ALD. A literature search showed that tantalum oxynitrides have superior dielectric properties compared to pure oxides [4], so experiments were performed with both pure $\mathrm{O}_{2}$ and $\mathrm{O}_{2} / \mathrm{N}_{2}$ mixtures. An Oxford Plasma Technologies RIE was used to oxidize the Ta surface. Temperature and gas composition were varied to yield a high value of capacitance and reasonable uniformity across the wafer. A mixture of $50 \% \mathrm{O}_{2}$ / $50 \% \mathrm{~N}_{2}$ and substrate temperature of $250{ }^{\circ} \mathrm{C}$ was found to give a repeatable value of $0.028 \mathrm{~F} / \mathrm{m}^{2}$.

\section{Inductors}

To achieve a large inductance and low resistance in a small area, thick $\mathrm{Cu}$ coils were electroplated on a temporary seed layer. Two photoresists were used: KMPR [5], a high aspect ratio negative resist, and $\mathrm{AZ} 4620$, a conventional positive thick resist. Although the KMPR yielded superior high aspect ratio plated structures (Fig. 1), difficulty removing the cross-linked KMPR caused us to ultimately revert to AZ 4620 which is easily removed in standard solvents and resist strippers.

\section{Fabrication Process Sequence}

The process is shown in cross-sections in Fig. 2. $100 \mathrm{~mm}$ glass wafers were used as substrates. A tri-layer stack of $\mathrm{Cr} / \mathrm{Au} / \mathrm{Ta}$ was sputtered and patterned using a liftoff technique (2a). This layer serves as the bottom conductor for the capacitors, and also to cross-under the inductor. The top Ta layer is plasma oxidized to form the capacitor dielectric $(2 \mathrm{~b})$. A contact mask is applied and the resultant $\mathrm{Ta}_{2} \mathrm{O}_{5}$ and Ta layers are plasma etched in the contact areas. A $2 \mu \mathrm{m}$ thick layer of positive photoresist is patterned and hard baked to form a sacrificial layer for creating an air-gap between the inductor and the cross-under layer (2c). A seed layer of $\mathrm{Ti} / \mathrm{Cu}$ is then 
sputtered everywhere as a plating base (2d). The plating resist mask is applied and patterned to define the spiral inductor and the top capacitor metal (2e). A commercial $\mathrm{Cu}$ bath is used to plate up 15 microns of $\mathrm{Cu}$, after which the resist plating mask, seed layer, and sacrificial photoresist layer are removed (2f). Finally the wafer is coated with photoresist and diced to the desired size. A completed resonator is shown in Fig. 3. A unit cell containing an array of resonators and test structures, coated in Agar gel is shown in Fig. 4. These arrays were further diced to provide individual resonators when required for in-vivo testing.

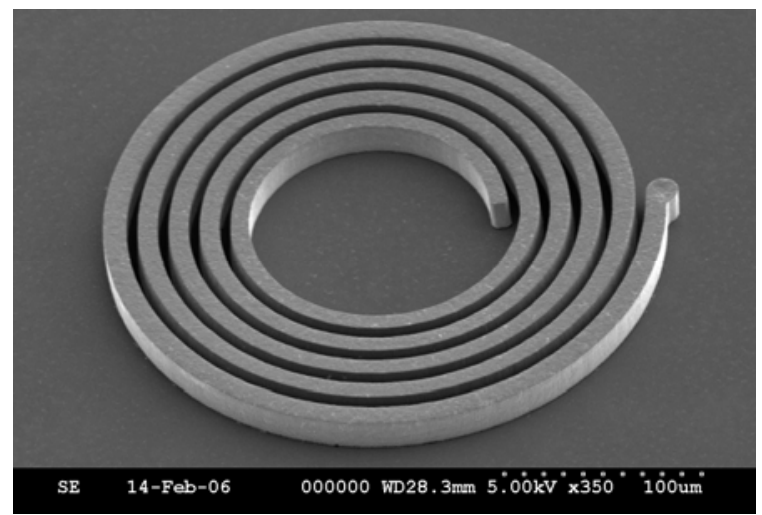

Figure 1. Electroplated Cu coil $15 \mu \mathrm{m}$ thick, plated through high aspect ratio KMPR negative resist. Coils are $10 \mu \mathrm{m}$ wide with a 5 um gap.
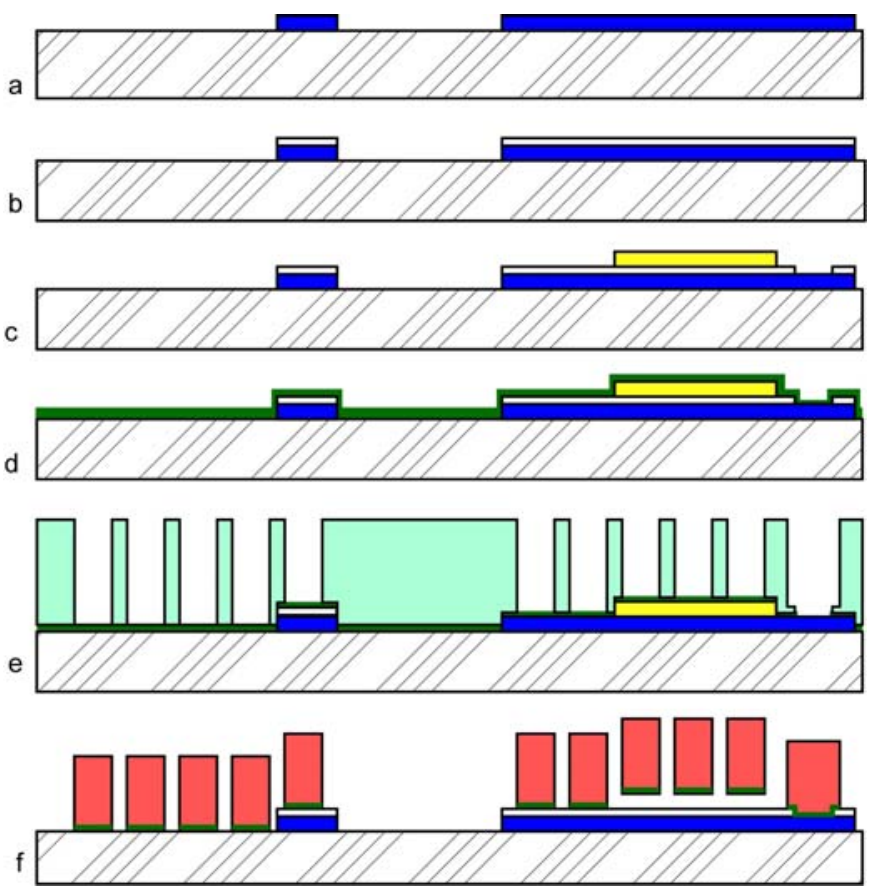

Figure 2. Fabrication sequence for the resonant micro-tags.

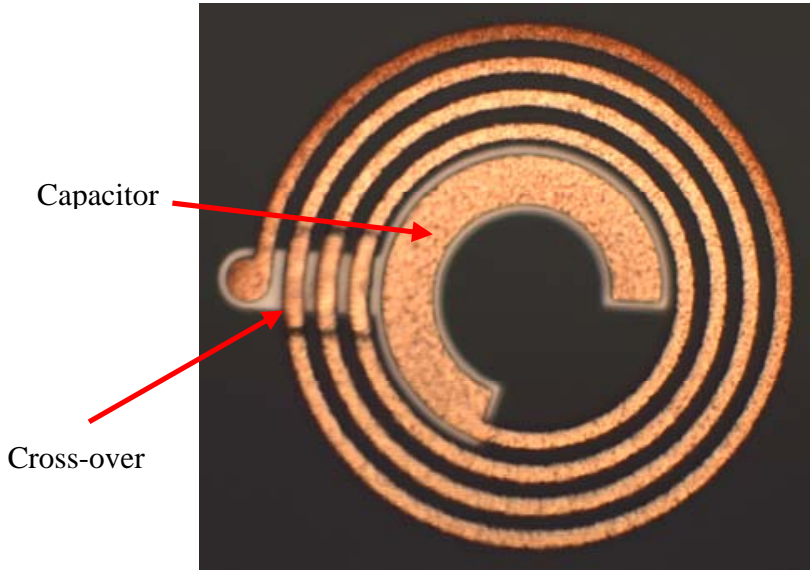

Figure 3. LC resonator with spiral inductor, air-bridge and capacitor. Device diameter is 370 microns.

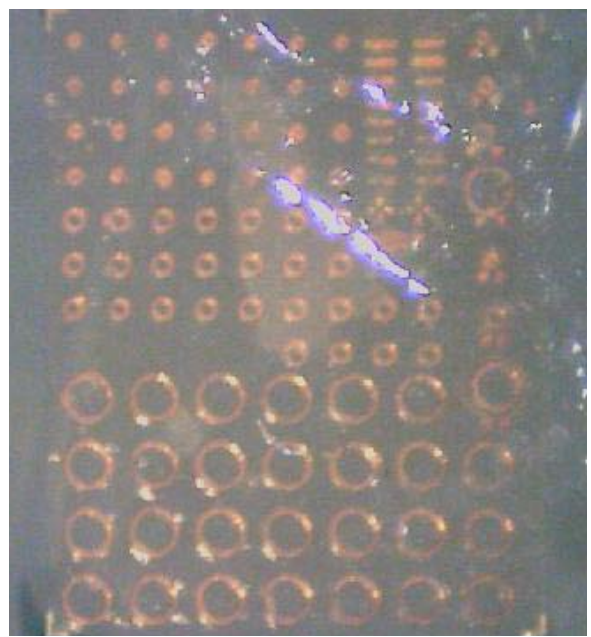

Figure 4. Array of resonators with 0.3, 0.5, and $1 \mathrm{~mm}$ diameters encapsulated in Agar gel.

\section{RESULTS}

Spiral Inductors

Test inductors included on the mask set were measured using an HP LCR meter. Measured vs. calculated values of inductance are shown in Fig. 5. The measured values are about $70-80 \%$ of the calculated values, perhaps due to stray capacitance and inductance of the test leads.

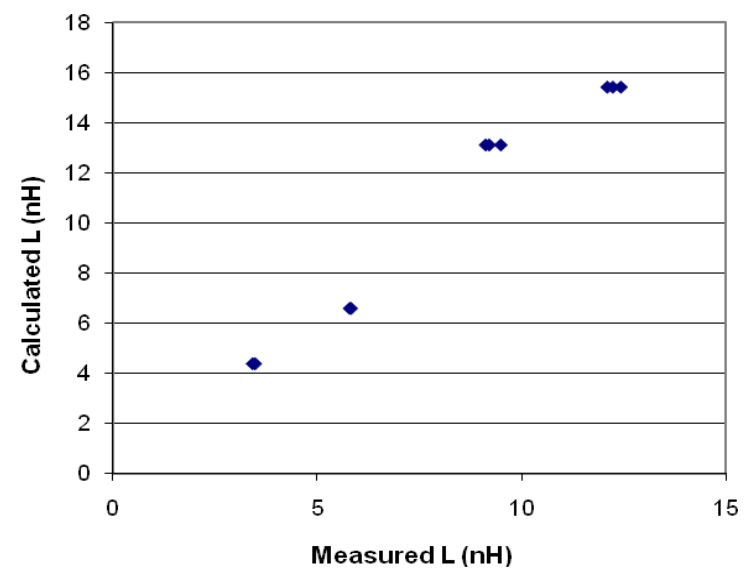

Figure 5. Measured vs. calculated inductances. 


\section{SCANNING RF MICROSCOPE AND MRI IMAGES}

A technique similar to that used in scanning near field microwave microscopy [6] was used in order to characterize the MRDs. We call this technique ultra high frequency scanning microscopy (UHF microscopy) because the frequency range of interest is typically 87- $207 \mathrm{MHz}$. This technique involves scanning a coil with a diameter of $1.5 \mathrm{~mm}$ (detection coil) that couples inductively to the MRDs without contacting them. The linear magnitude and phase of the reflection coefficient $\left(\mathrm{S}_{11}\right)$ measured by a network analyzer (HP 8751A) is stored along with the spatial coordinates using custom software. By plotting the $S_{11}$ coefficient as a function of position an RF image of the MRDs can be obtained for a specific frequency (Fig. 6). A good correlation was obtained between the location of MRDs in MRI spin echo image (Fig 8a) and the UHF microscopy image (Fig 8b). A good concordance between MRI and UHF microscopy can be seen for 300 and 500 microns resonators.

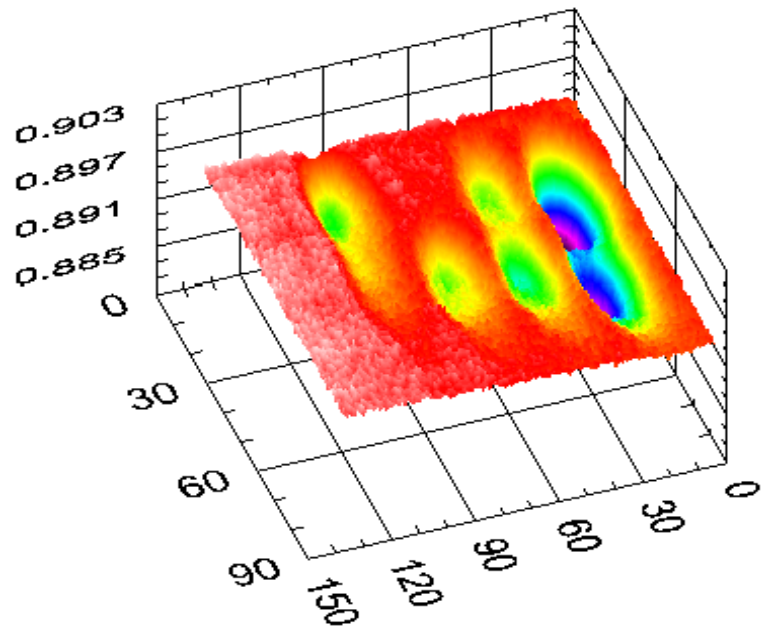

Figure 6. Scanning UHF image $\left(S_{11}\right)$ of array of MRD's.

The variation of $S_{11}$ recorded during UHF microscopy experiment is analyzed as a function of frequency for a given $\mathrm{x}$, $\mathrm{y}$ position and, as a result, the frequency response of MRD is obtained. To understand these variations and to predict which MRDs can be visualized in MRI experiments we developed an analytical model. This model is based on the analogy between UHF microscopy and Radio Frequency Identification (RFID) systems. The detection coil and MRDs are similar to the transmitter coil and the transponder in an RFID system. In an RFID system, mutual inductance between the readout coil and the LC circuit of the tag allows readout. Based on this analogy, we can use the relationship derived by Finkenzeller [7] for the complex transformed impedance $\mathrm{Z}_{\mathrm{T}}^{\prime}$ of MRDs "seen" in detection circuit:

$$
Z_{T}^{\prime}=\frac{\omega^{2} k^{2} L_{1} L_{2}}{R_{2}+j \omega L_{2}+Z_{2}}
$$

where $L_{1}$ and $L_{2}$ are inductances of detection and MRDs coils, respectively; $\omega$ is angular frequency; $R_{2}$ is the $M R D$ coil resistance, $Z_{2}$ is the MRD non-inductive impedance (given by Equation 3) ; $k$ coupling coefficient [7] was computed as:

$$
k(x)=\frac{r_{M R D}^{2} r_{\text {reader }}^{2}}{\sqrt{r_{M R D} r_{\mathrm{det}}}\left(\sqrt{x^{2}+r_{\mathrm{det}}^{2}}\right)^{3}}
$$

where $r_{M R D}$ and $r_{\text {det }}$ are the average radius of MRD and detection coils, respectively placed at distance $x . Z_{2}$ impedance from Equation 1 is associated with the capacitor $C_{2}$ made up of a parallel tuning capacitor $\left(C_{T}\right)$ and a parasitic capacitance $\left(C_{P}\right)$ from the real circuit. A load resistor $\left(R_{L}\right)$ that describes the current consumption in the RFID circuit was also considered, which could represent leakage current (if any) through the dielectric. In these conditions, the MRD non-inductive impedance becomes:

$$
Z_{2}=\frac{R_{L}}{1+j \omega R_{L} C_{2}}
$$

MEMS chips were attached on a microscope slide, immersed in a Phosphate-Buffer Saline (aqueous media supplemented with $150 \mathrm{nmM} \mathrm{NaCl}$ ) and imaged in a 3T MRI scanner (GE) using an 8 - leg birdcage coil $(110 \mathrm{~mm}$ in length and $60 \mathrm{~mm}$ in diameter). Fig. 7 is a spin-echo image with the following experimental MRI parameters: repeat time - TR=300 $\mathrm{ms}$; echo time $-\mathrm{TE}=10 \mathrm{~ms}$; the field of view $(\mathrm{FOV})$ was $80 \mathrm{~mm}$ $\times 80 \mathrm{~mm}$ with a 256 by 128 data matrix.

Relatively B1 sensitive spin-echo sequences showed good MRD contrast, whereas relatively B1 insensitive gradient echosequences showed none, demonstrating that the MRD contrast mechanism is due to perturbation of the $\mathrm{B} 1$ field.

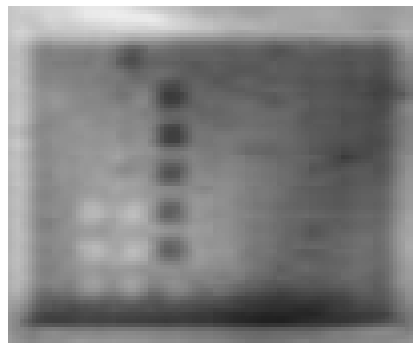

Figure 7. MRI image of MRD array.

Computed and experimentally determined frequency responses for an MRD in are shown in Figure 8c. The analytical model and experimental data show that a quality factor of 7.2 was measured for this device. The frequency analysis of MRDs based on UHF microscopy allowed us to select the devices that will be visualized in MRI experiments. MRD active devices contain the Larmor frequency 127.7 MHz (green line in Figure 8c) within their $3 \mathrm{~dB}$ region.

Two active and two non-active MRDs were subcutaneously implanted in a mouse flank (Fig. 9). The MicroCT image (InVeon, Siemens Medical Solutions) shows that at least 3 MRDs are placed in the same plan (Fig. 9C). These devices have been imaged using MRI in a clinical 3T scanner (Fig. 9b). The magnetic field generated by the MRDs was mapped (Figure 9a) using an algorithm [8] that considers two gradient - echo scans at flip angles of 30 degrees and 60 degrees. These pictures show that only the active devices have been located due to their self generated magnetic field. The maximum B1value determined from MRD (labeled 1 in Figure 8) was $8.610^{-7}$ Tesla. 


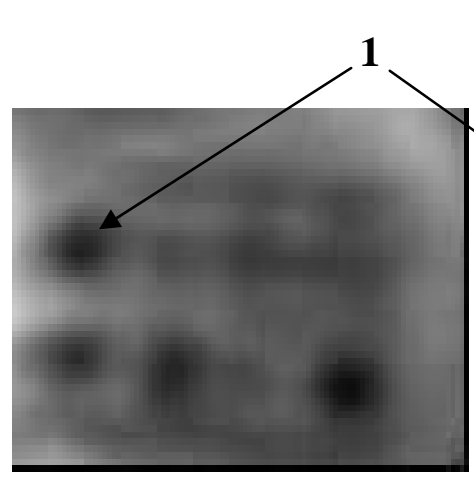

a. MRI

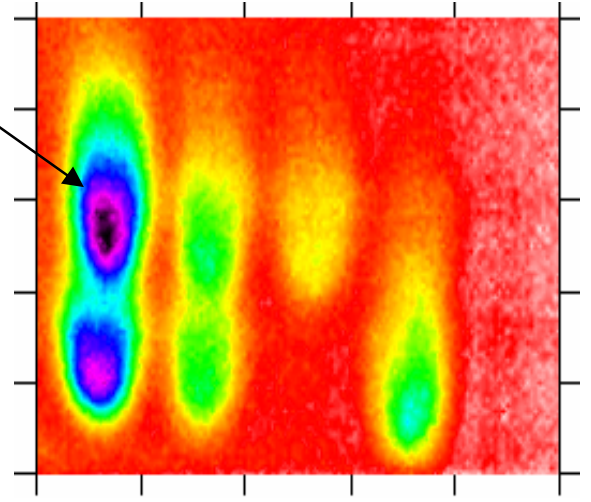

b. UHF scanning microscopy

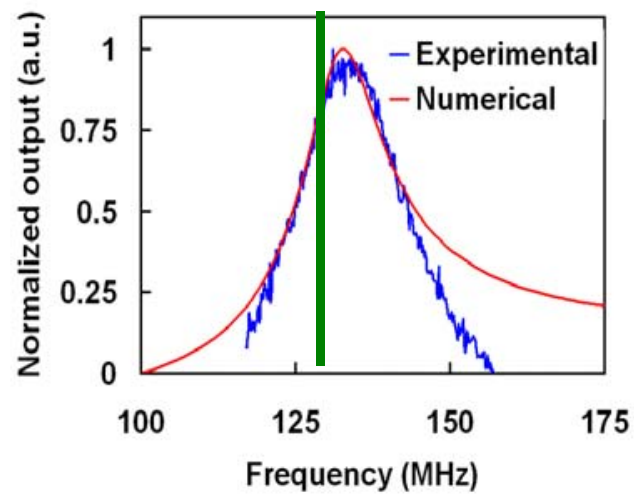

c. LC resonance curve

Figure 8. Comparison of MRI image (a) and UHF scanning image (b). Measured LC resonance with peak near $127.7 \mathrm{MHz}$ (green line).

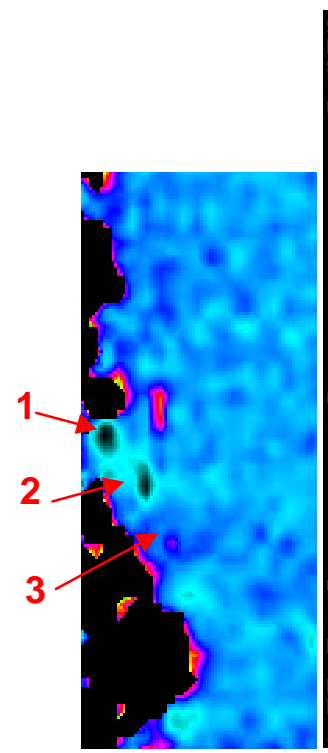

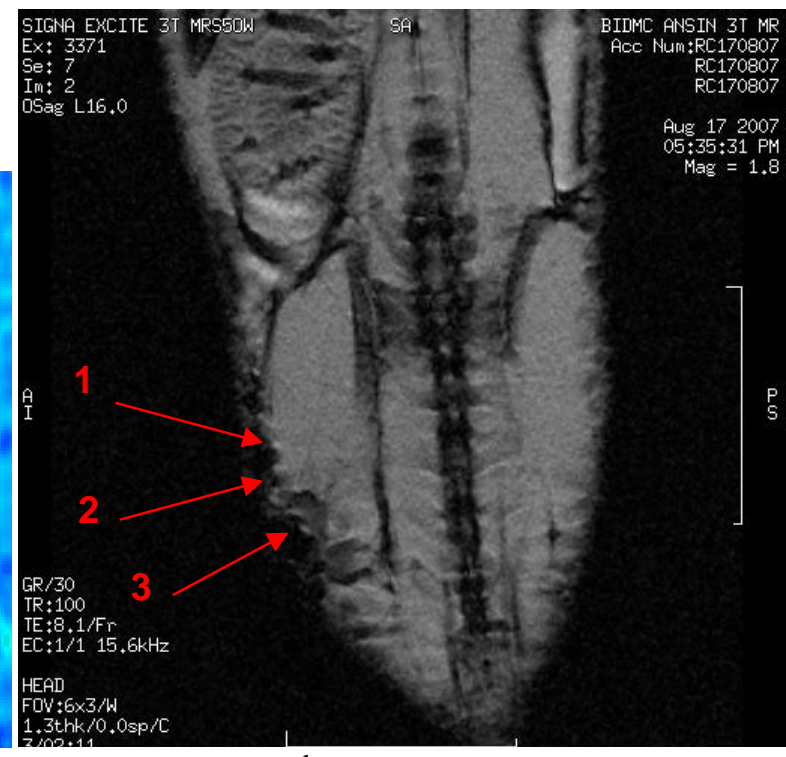

b.

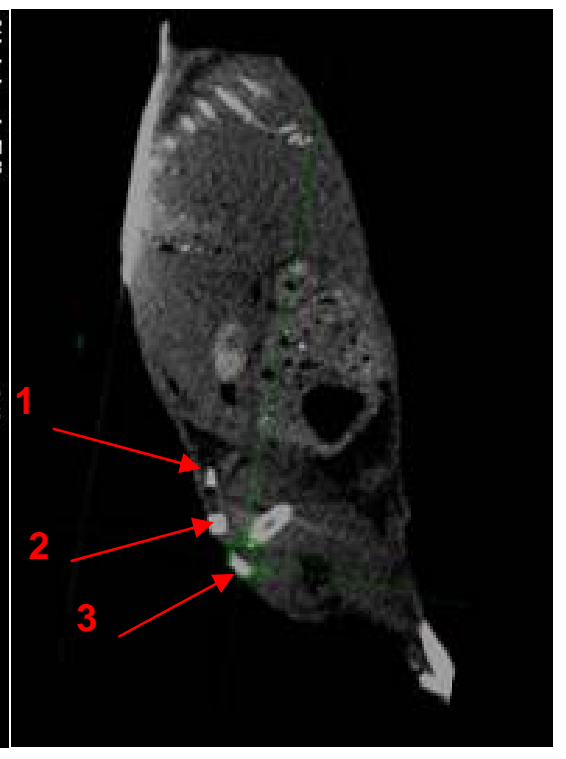

Fig. 9. Comparison of MRI B1 magnetic field map (a), MRI image (b) and micro-CT image (c). Devices are implanted in a mouse flank.

\section{CONCLUSIONS}

We have demonstrated that micro-solid-state resonant devices are capable of being imaged in vivo and in vitro using a clinical $3 \mathrm{~T}$ MRI, and that they can serve as B1 contrast agents. A novel plasma oxidation process was utilized to fabricate high value capacitance in a small area. A novel UHF microscope was built to image the devices. All types of MRDs (300, 500 and 1000 microns) designed and manufactured were detected in clinical 3T MRI. The scanning time was less than 10 minutes for each scan.

Future studies will focus on miniaturizing these devices to the cellular scale, on generating contrast at radiofrequencies not present in the human body, and on probing the limits of MRI sensitivity.

\section{ACKNOWLEDGEMENTS}

Supported by an Application Development award from the Center for the Integration of Medicine and Innovative Technology (CIMIT), US Army DAMD17-02-2-0006.

\section{REFERENCES}

[1] M. H. Levitt, "Spin Dynamics", pp. 315-335, (Wiley and Sons, 2001).

[2] Feridex and Combidex are products of Advanced Magnetics: http://www.amagpharma.com/.

[3] S.S. Mohan et. al., "Simple Accurate Expressions for Planar Spiral Inductances", IEEE Journal of Solid-State Circuits, Vol. 34, No. 10, October 1999, pp. 1419-1424.

[4] H. Jung, K. Im, D. Yang, and H. Hwang "Electrical and Reliability Characteristics of an Ultrathin TaOxNy Gate Dielectric Prepared by $\mathrm{ND}_{3}$ Annealing of $\mathrm{Ta}_{2} \mathrm{O}_{5}$ " IEEE ELECTRON DEVICE LETTERS, VOL. 21, NO. 12, DECEMBER 2000, pp. 563-565.

[5] KMPR photoresist, MicroChem Inc.: http://www.microchem.com/products/kmpr.htm

[6] R. Ciocan and N. Ida, IEEE Transactions on Magnetics, 40, 651 (2004).

[7] K. Finkenzeller "RFID Handbook Fundamentals and Applications in Contactless SmartCards and Identification", (John Wiley \& Sons Inc New York, 2003)., pp 88-91.

[8] E.K. Insko and L. Bolinger, Journal of magnetic resonance, series A, 103, 82(1993). 